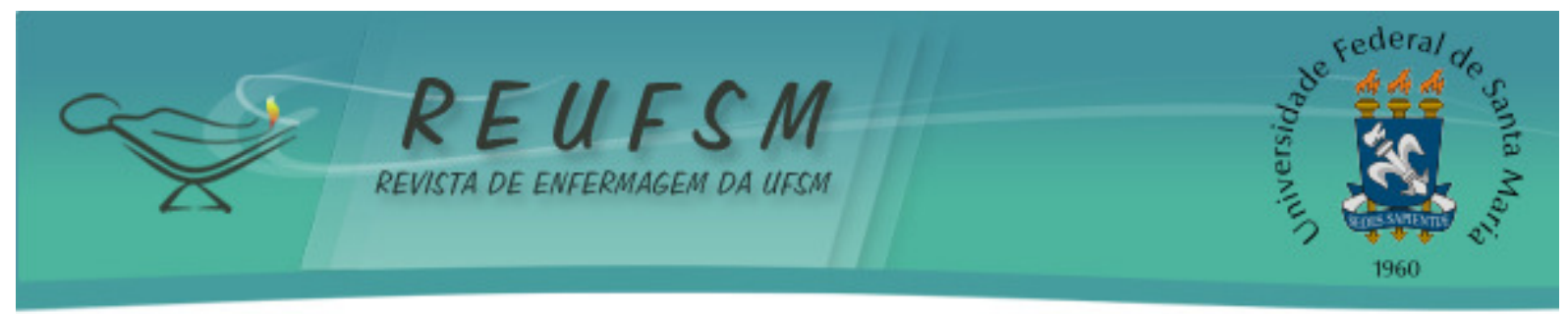

ARTIGO ORIGINAL

\title{
NÍVEL DE COMPLEXIDADE ASSISTENCIAL E DIMENSIONAMENTO DE ENFERMAGEM NO PRONTO-SOCORRO DE UM HOSPITAL PÚBLICO
}

\section{COMPLEXITY LEVEL OF ASSISTANCE AND NURSING SIZING IN A EMERGENCY UNIT OF A PUBLIC HOSPITAL}

\section{LA COMPLEJIDAD ASISTENCIAL Y LA DIMENSIÓN DE ENFERMERÍA EN UN SERVICIO DE URGENCIA}

\author{
Ana Cristina Geiss Casarolli ${ }^{1}$ \\ Thaís Dresch Eberhardt² \\ Anair Lazzari Nicola ${ }^{3}$ \\ Luciana Magnani Fernandes ${ }^{4}$
}

Doi: $10.5902 / 2179769216811$

RESUMO: Objetivo: analisar o nível de complexidade assistencial e o dimensionamento dos profissionais de enfermagem no pronto-socorro de um hospital público. Método: estudo transversal descritivo realizado com dados extraídos de prontuários de pacientes. Resultados: foram avaliados 998 prontuários, destes houve predomínio $(73,3 \%)$ de pacientes classificados em cuidados mínimos. Em relação aos profissionais de enfermagem, o quadro existente por turno de trabalho correspondeu a $32,6 \%$ para a categoria enfermeiro e $91,5 \%$ para auxiliares/técnicos de enfermagem do quadro de pessoal projetado para a unidade. Conclusões: o predomínio de pacientes classificados como em cuidados mínimos pressupõe a inadequação do fluxo de atenção dos serviços de saúde, e consequente utilização equivocada da atenção terciária, gerando número expressivo de atendimentos. Este contexto é agravado pelo quantitativo reduzido de profissionais de enfermagem para prestar assistência com qualidade e segurança.

Descritores: Enfermagem; Serviços médicos de emergência; Downsizing organizacional; Equipe de enfermagem; Gestão em Saúde.

ABSTRACT: Aim: to assess the level of care complexity and the dimensioning of the nursing staff attending in the emergency unit of a public hospital. Method: descriptive and documental study, performed with data extracted from patients' records. Results: we assessed 998 records. There was a prevalence $(73,3 \%)$ of patients requiring minimal care. Concerning the nursing staff, the situation according to work shifts corresponded to $32,6 \%$ for nurses and $91,5 \%$ for nursing auxiliary/technicians from the frame projected for the unit. Conclusions: the prevalence of patients needing minimal care may stem from an inadequate flow of care provided for the health services and, consequently, producing an unnecessary use of the tertiary health services and, so, increasing the number of

\footnotetext{
1 Enfermeira, Especialista em Gerenciamento de Enfermagem em Clínica Médica e Cirúrgica, Mestranda do Programa de Pós-Graduação em Enfermagem, Universidade Federal de Santa Maria, Santa Maria, Rio Grande do Sul, Brasil. E-mail: anacasarolli@hotmail.com.

${ }^{2}$ Enfermeira, Especialista em Gerenciamento de Enfermagem em Clínica Médica e Cirúrgica e em Acupuntura, Mestranda do Programa de Pós-Graduação em Enfermagem, Universidade Federal de Santa Maria, Santa Maria, Rio Grande do Sul, Brasil. E-mail: thaiseberhardt@gmail.com.

${ }^{3}$ Enfermeira, Doutora em Enfermagem, Docente do Colegiado de Enfermagem da Universidade Estadual do Oeste do Paraná, Cascavel, Paraná, Brasil. E-mail: anairln@yahoo.com.br.

${ }^{4}$ Enfermeira, Doutora em Enfermagem, Docente do Colegiado de Enfermagem da Universidade Estadual do Oeste do Paraná, Cascavel, Paraná, Brasil. E-mail: lumagna@terra.com.br.
} 


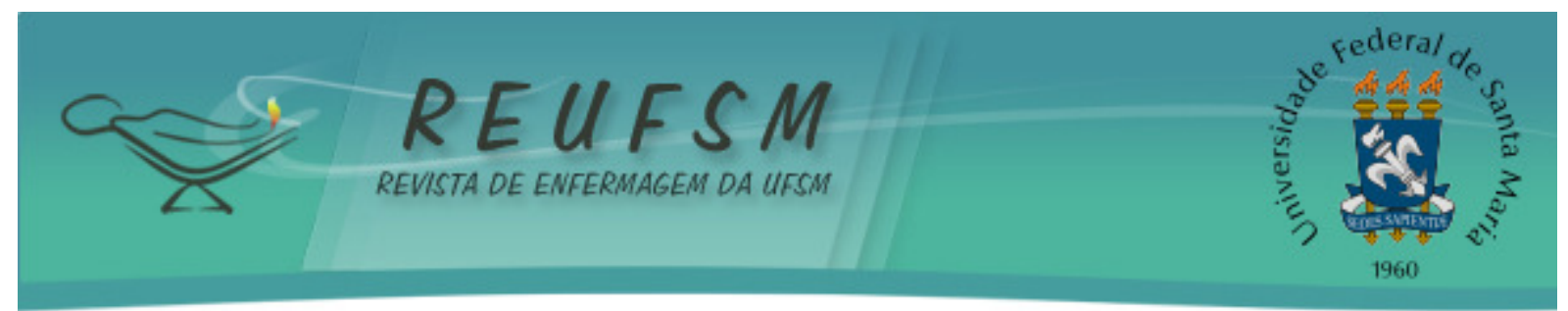

attendances. This context is worsened by the reduced number of nursing workers available to provide a qualified and safe care.

Descriptors: Nursing; Emergency medical services; Personnel downsizing; Nursing, team; Health Management.

RESUMEN: Objetivo: investigar la complejidad asistencial y la dimensión del profesional de enfermería en el sector de urgencias de un hospital público. Método: estudio descriptivo y documental realizado con datos de registros de los pacientes. Resultados: fueron evaluados 998 registros, en que hubo el predominio $(73,3 \%)$ de pacientes clasificados en cuidados mínimos. En relación a los profesionales de enfermería, el cuadro existente por turno de trabajo correspondió a 32,6\% para la categoría de enfermero y $91,5 \%$ para auxiliares/técnicos de enfermería del cuadro de profesionales proyectado para la unidad. Conclusiones: el predominio de pacientes clasificados como cuidados mínimos presupone la inadecuación del flujo de atención de los servicios de salud y consecuente uso equivocado de la atención terciaria, generando número expresivo de atendimientos. Este contexto se agrava por la reducida cantidad de profesionales de enfermería para prestar asistencia con calidad y seguridad.

Descriptores: Enfermería; Servicios médicos de urgencia; Reducción de personal; Grupo de enfermería; Gestión en Salud.

\section{INTRODUÇÃO}

A Política Nacional de Atenção Básica é concebida como estratégia para responder, de forma contínua e regionalizada, grande parte das necessidades de saúde, integrando ações preventivas e curativas ao indivíduo e à comunidade. ${ }^{1}$ Contudo, o esforço no incremento das ações básicas de saúde não tem sido suficiente para resolver os problemas de saúde da população no nível primário de atenção. ${ }^{2}$

Apesar dos avanços no processo de implementação do Sistema Único de Saúde (SUS), torna-se ainda evidente a necessidade de superar a fragmentação das ações e serviços de saúde e qualificar a gestão do cuidado. Para isso, existe a proposta de organizar e implantar as Redes de Atenção à Saúde (RAS), por meio de algumas estratégias, como a Rede de Atenção às Urgências e Emergências. ${ }^{3}$

Nesta proposta, a estrutura organizacional a ser utilizada é a de que os usuários com quadros agudos possam ser atendidos por todas as portas de entrada do SUS, possibilitando a resolução do problema ou transferindo-o para outro serviço de maior complexidade tecnológica, considerando um sistema hierarquizado, conforme institui a Política Nacional de Atenção às Urgências. ${ }^{3}$

Nessa estrutura, os prontos-socorros, além de assegurar as manobras de sustentação de vida em casos de urgência e emergência, representam a porta de entrada para os usuários com queixas crônicas e sociais, indivíduos vítimas de violências e com doenças crônico-degenerativas. ${ }^{4}$

Considerando o perfil epidemiológico variável de atendimento nos prontossocorros, as ações de cuidado devem ser centradas nas necessidades da população, da atenção contínua e integral e pelo cuidado multidisciplinar. ${ }^{5}$ Nesta unidade, o enfermeiro deve possuir habilidades e conhecimentos específicos para articular ações de gerência da unidade, dos recursos humanos e materiais e do cuidado prestado, para atender com qualidade e segurança os pacientes. ${ }^{6}$

Para o desenvolvimento da assistência com qualidade e segurança, há a necessidade de provisão adequada de recursos, principalmente de profissionais de enfermagem. 0 dimensionamento destes profissionais é foco da atenção dos enfermeiros e 


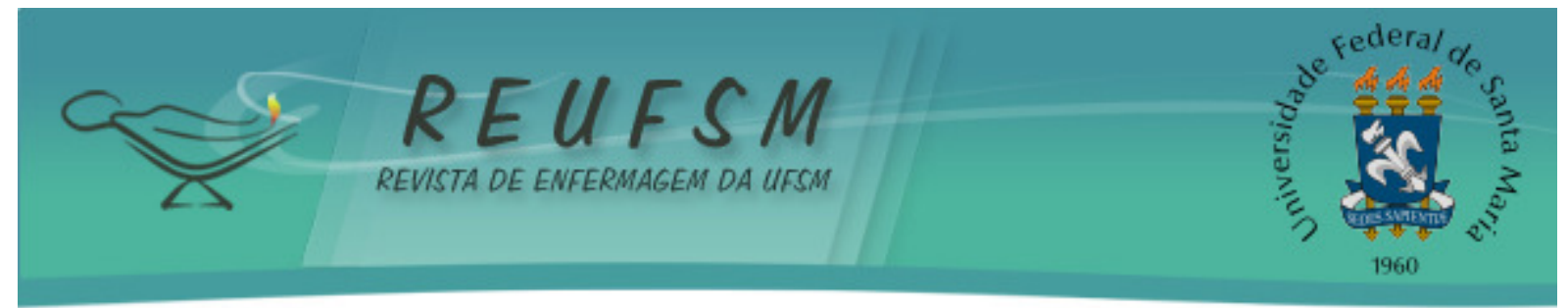

dos administradores dos serviços de saúde, por interferir diretamente na assistência de enfermagem. ${ }^{4}$

O dimensionamento do quadro de profissionais de enfermagem é determinado pelo contexto da unidade e pela identificação da carga de trabalho. Para identificar essas variáveis, faz-se necessário medir o tempo que a enfermagem utiliza para prestar a assistência. Para isso, são utilizados Sistemas de Classificação de Pacientes (SCP) a fim de identificar o tempo de cuidado da enfermagem para cada categoria de cuidado. ${ }^{7}$

Estabelecer, sob o enfoque quantitativo e qualitativo, o quadro de profissionais de enfermagem necessário para atender a necessidade de assistência, é de responsabilidade do enfermeiro, com base nas normas técnicas estabelecidas pela Resolução do Conselho Federal de Enfermagem (COFEN) $n^{\circ} 293$ de 2004. ${ }^{8}$ Esta Resolução estabeleceu parâmetros para dimensionar o quantitativo de pessoal da enfermagem nas instituições de saúde e recomenda que o dimensionamento seja realizado considerando-se as características da instituição, a assistência prestada e o perfil da clientela.

A aplicação da metodologia dimensionamento de pessoal de enfermagem, proposta por esta resolução, pode contribuir para reduzir as dificuldades encontradas pelos enfermeiros no gerenciamento da assistência, deixando de ser uma análise subjetiva do quantitativo de profissionais necessários para uma assistência segura e de qualidade.

Desse modo, este estudo teve como objetivo analisar o nível de complexidade assistencial e o dimensionamento dos profissionais de enfermagem do pronto-socorro de um hospital público.

\section{MÉTODO}

Trata-se de um estudo transversal descritivo, desenvolvido por meio de pesquisa documental, com abordagem quantitativa, realizado no pronto socorro de um hospital universitário do Estado do Paraná.

O pronto socorro possui oito leitos para internamento, divididos em Sala de Emergência e internamento/observação. A Sala de Emergência recebe pacientes graves, com risco de morte, provindos da Unidade de Pronto Atendimento (UPA), Serviço de Atendimento Móvel de Urgência (SAMU), Serviço Integrado de Atendimento ao Trauma em Emergência (SIATE) e pacientes transferidos de hospitais da região.

Já a unidade de internamento/observação admite pacientes sem risco de morte, advindos dos serviços acima citados e atende a demanda espontânea. Os pacientes são admitidos após passar por atendimento médico e de enfermagem e podem permanecer em observação ou internados. Quando internados, permanecem no setor até que possam ser transferidos para os demais setores hospitalares. Entretanto, quando não há vagas disponíveis o paciente pode permanecer no setor de internamento/observação do pronto socorro até receber alta médica.

Foram incluídos todos os pacientes atendidos e/ou internados na unidade de internamento do pronto-socorro no período de coleta de dados que foi de 30 dias, entre junho e julho de 2012, durante os períodos matutino e vespertino. 0 recorte temporal justifica-se por ser o período mínimo para que se obtenha uma amostragem que reflita o perfil dos pacientes atendidos. ${ }^{7}$

A coleta de dados foi realizada nos prontuários eletrônicos dos pacientes submetidos a atendimento médico. 0 sistema eletrônico contém também o instrumento de classificação de pacientes proposto por Fugulin ${ }^{9}$ para pacientes adultos e o proposto por Dini $^{10}$ para pacientes pediátricos. Estes instrumentos são preenchidos diariamente pelo enfermeiro da unidade. 


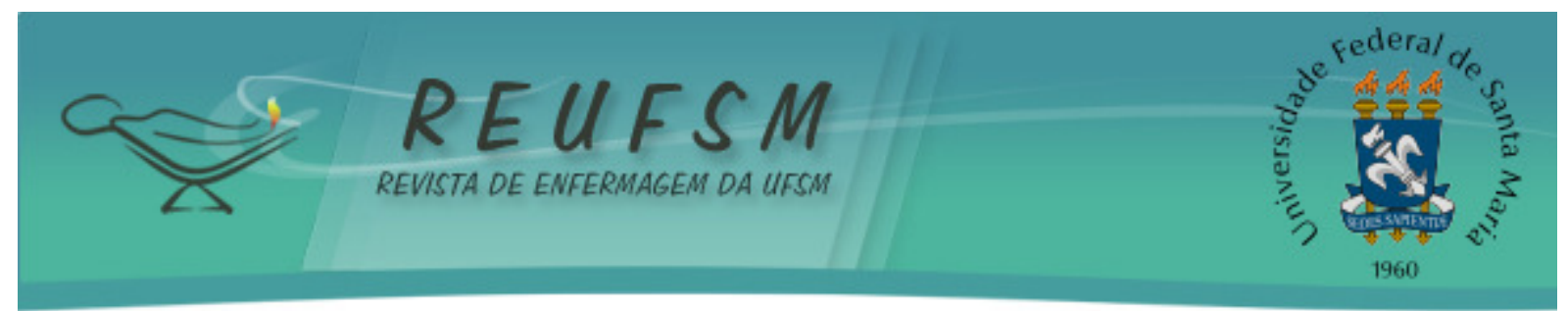

As variáveis em estudo foram idade, sexo e o escore final do SCP. Os instrumentos de Fugulin ${ }^{9}$ e Dini ${ }^{10}$ estabelecem para cada área do cuidado, pontuações que variam de um a quatro, de acordo com a complexidade assistencial sendo um a pontuação correspondente à menor complexidade e quatro à maior complexidade. No final da avaliação, realizou-se a soma das pontuações e verificou-se o grau de dependência assistencial do paciente. Os pacientes que receberam atendimento médico e não permaneceram em observação foram classificados como cuidado mínimo, uma vez que estes pacientes receberam cuidado de enfermagem de baixa complexidade.

Os dados referentes ao quantitativo de profissionais de enfermagem foram coletados da planilha de gerenciamento do Serviço de Enfermagem preenchido diariamente pelo enfermeiro da unidade. Para o cálculo da carga horária de assistência de enfermagem e número necessário de profissionais de enfermagem, foi utilizada a resolução do COFEN $n^{\circ}$ $293 / 2004^{8}$. De acordo com essa resolução, para efeito de cálculo, devem ser consideradas horas de enfermagem por leito nas 24 horas, 3,8 horas de enfermagem por cliente na assistência mínima; 5,6 horas na assistência intermediária; 9,4 horas na assistência semiintensiva; e 17,9 horas na assistência intensiva ${ }^{8}$.

A distribuição percentual do quadro de profissionais de enfermagem obedeceu às proporções, também estabelecidas pela Resolução COFEN $n^{\circ} 293 / 04^{8}$. A carga horária de trabalho semanal da equipe de enfermagem do presente estudo era de 36 horas, distribuídas em plantões de seis horas diárias, com uma folga semanal, para o período diurno. Para o período noturno, os plantões são de 12 horas com 60 horas de descanso, sendo que esta modalidade não dá direito a folgas semanais.

Para análise e apresentação dos dados referentes às variáveis, foi realizada estatística descritiva simples (frequência absoluta e relativa).

O estudo foi desenvolvido após a aprovação pelo Comitê de Ética em Pesquisa institucional, sob parecer $n^{\circ} 014 / 2011$, respeitando-se os aspectos éticos para a pesquisa com seres humanos.

\section{RESULTADOS}

Foram avaliados 998 prontuários. Do total de pacientes, $130(13,0 \%)$ foram submetidos apenas à consulta médica, exames e/ou administração de medicamentos. A média diária de pacientes foi de 33,3 pacientes, sendo 28,3 de adultos/idosos e 5,0 de crianças/adolescentes.

Em relação à caracterização dos pacientes, 500 (57,6\%) eram do sexo masculino e $368(42,4 \%)$ do sexo feminino, $849(85,1 \%)$ eram adultos e idosos e $149(14,9 \%)$ crianças e adolescentes na faixa etária de zero a 13 anos incompletos.

Quanto ao grau de dependência, obteve-se um total de $731(73,3 \%)$ pacientes classificados em cuidados mínimos; 203 (20,3\%) em cuidados intermediários; 63 (6,3\%) em cuidados de alta dependência e $01(0,1 \%)$ em cuidados semi-intensivos.

Em relação à média diária de pacientes adultos/idosos, aqueles classificados em cuidados mínimos foram 22,7; cuidados intermediários 3,9; cuidados de alta dependência 1,7. A média diária de crianças/adolescentes de cuidados mínimos foi 1,7; cuidados intermediários 2,9; cuidados de alta dependência 0,4.

Para a assistência mínima observou-se uma média diária de horas de assistência de enfermagem de 92,6 horas; para a assistência intermediária, 37,9 horas; para a assistência semi-intensiva 20,0 horas. Isto gerou, para o profissional enfermeiro, uma carga horária de 51,5 horas de assistência diária e 99,1 horas para técnicos e auxiliares de enfermagem. 


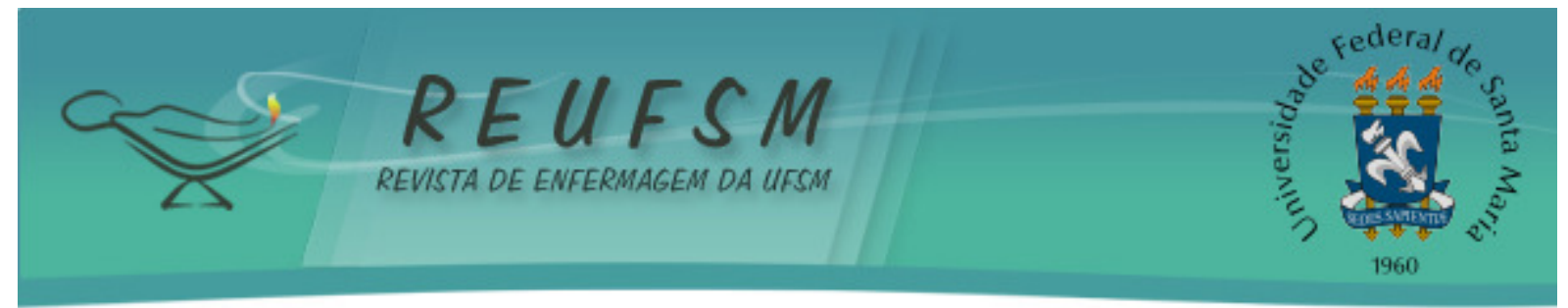

No que se refere ao quadro de pessoal existente, obteve-se a média diária de 2,8 enfermeiros e 15,1 técnicos de enfermagem. Ao consideramos o quadro de profissionais por turno de trabalho, observou-se 0,9 enfermeiro e 5,0 técnicos e auxiliares de enfermagem.

Para o quadro recomendado, sem considerar o quadro de pessoal para cobertura de faltas previstas (férias e licenças) e não previstas eram necessários 8,6 enfermeiros e 16,5 técnicos e auxiliares de enfermagem para atender a demanda da unidade nas 24 horas, sendo 2,9 enfermeiros e 5,5 técnicos de enfermagem por turno de trabalho.

\section{DISCUSSÃO}

Estudo desenvolvido sobre a caracterização do perfil de pacientes atendidos na unidade de pronto socorro, mostra dados semelhantes com $73,2 \%$ dos pacientes caracterizados por complexidade assistencial mínima, ${ }^{4}$ definido como sendo um paciente estável sob o ponto de vista clínico e de enfermagem e autossuficiente em relação ao atendimento das necessidades. ${ }^{8}$

Além de atendimentos em casos em que o paciente tem risco de vida e aqueles que permanecem em observação, a equipe da unidade divide-se ainda para prestar assistência aos pacientes que necessitam de consulta médica, procedimentos de enfermagem, exames e medicações, o que correspondeu a $13,03 \%$ do total de pacientes atendidos na unidade de internamento e observação do pronto socorro.

Mesmo sem a efetivação da internação desses pacientes, os atendimentos sobrecarregavam a unidade e a equipe multiprofissional que atuava nesse serviço, incluindo a equipe de enfermagem pela existência de um grande número de pacientes classificados como cuidados mínimos e não internados, requeriam assistência. Temos como exemplo os que eram atendidos nos consultórios médicos, alguns procedimentos ambulatoriais em que o paciente dava entrada pelo pronto-socorro, administração de medicações, pacientes que requeriam inalação, exames laboratoriais e de imagem. $\mathrm{Na}$ avaliação do quadro de pessoal de enfermagem comparativo, verificamos que o quantitativo de profissionais existente na unidade não corresponde com o projetado, segundo o método utilizado e as horas médias de assistência preconizadas pela Resolução COFEN n ${ }^{\circ} 293 / 04$.

Em relação à categoria enfermeiro, o quadro existente por turno de trabalho, correspondeu a $32,6 \%$ do quadro de pessoal projetado para a unidade. Para o quadro de técnicos e auxiliares de enfermagem observou-se $91,5 \%$ do quantitativo necessário para a assistência diária.

Diante do quantitativo reduzido, mais evidenciado quando se trata do profissional enfermeiro, pressupõe-se que este profissional possa ter dificuldade em desempenhar todas as atividades que são de sua competência técnica e científica, sendo necessário delega-las para os técnicos e auxiliares de enfermagem. Vale ressaltar que de acordo com a lei do exercício profissional, o enfermeiro exerce privativamente algumas atividades, dentre as quais pode ser destacada os cuidados de enfermagem de maior complexidade técnica e que exijam conhecimentos de base científica e capacidade de tomar decisões imediatas. $^{11}$

A maioria dos pacientes fica acomodada em macas, nos corredores e sem privacidade e, muitas vezes, sem a presença do acompanhante, com recurso físico inadequado para atendimento, causando insatisfação ao paciente e à equipe que presta assistência. Essa problemática é agravada pelo quantitativo reduzido de profissionais de enfermagem no setor para prestar assistência com qualidade à alta demanda. Nessas condições, esses indivíduos tornam-se mais suscetíveis a complicações como queda, 


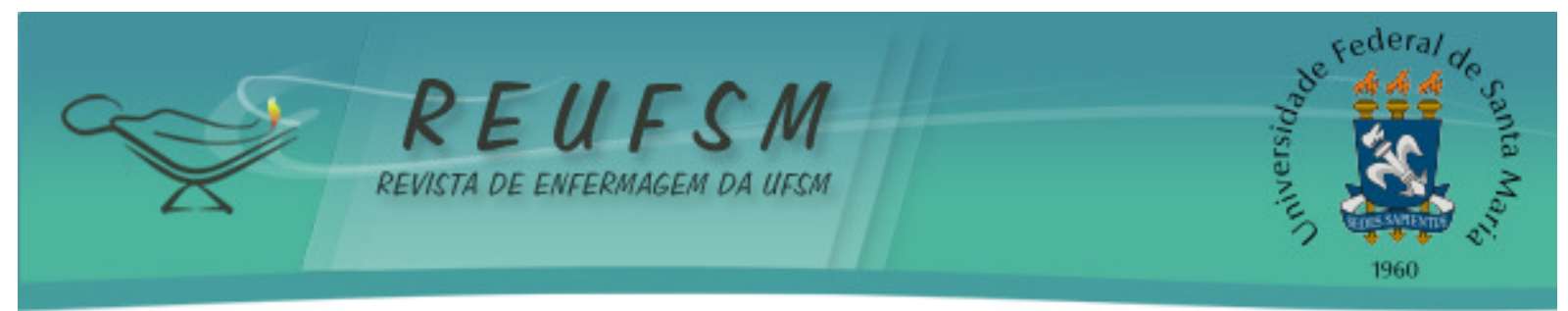

infecção relacionada à assistência à saúde, erro na administração de medicamentos e lesões de pele.

Estudo realizado com o objetivo de analisar a influência do dimensionamento do pessoal de enfermagem intensivista na qualidade do cuidado ao paciente crítico adulto, identificou a relação entre o sub-dimensionamento de trabalhadores de enfermagem e o aumento de taxas de infecção, de mortalidade, quedas, pneumonia associada à ventilação mecânica, extubação acidental e tempo de internação. ${ }^{12}$

Em estudo com o objetivo de identificar a concepção da equipe de enfermagem sobre possíveis riscos à segurança do paciente internado em unidade clínica, a carga de trabalho excessiva e o dimensionamento de pessoal de enfermagem insuficiente foram mencionados como sendo riscos à segurança dos pacientes. ${ }^{13}$

A realidade do pronto-socorro corrobora com pesquisas realizadas a nível nacional, na qual ficou evidenciado que também, os pacientes permaneciam em macas pelos corredores, sendo submetidos a condições insatisfatórias, uma vez que ficavam acomodados em locais de grande circulação de pessoas, alto índice de ruídos e luminosidade constante e, para a equipe multiprofissional, que acabava por desenvolver seu processo de trabalho em locais inapropriados para realização de assistência adequada. ${ }^{4,14}$

Os prontos-socorros são organizados para disponibilizar atendimento imediato, além de facilidades na realização de procedimentos diagnósticos e terapêuticos, configurando-se, muitas vezes, como a principal referência da população em busca de assistência médica. ${ }^{15}$

Apesar das dificuldades da rede básica de saúde em garantir acesso e resolutividade, parte considerável da população recorre ao pronto-socorro devido à legitimidade desse serviço perante os usuários. Essa população busca, prioritariamente, o serviço hospitalar devido à confiança nos profissionais, expectativas sobre a qualidade da assistência, experiência pessoal e/ou da rede social, satisfação com o atendimento e a acessibilidade geográfica. $^{2}$

As diferenças entre a lógica da população e a organização do sistema de saúde e vários outros fatores, determinam a utilização inadequada dos serviços de saúde, gerando número expressivo de atendimentos que poderiam ser resolvidos na rede básica de saúde. No entanto, a única alternativa para o atendimento efetivo nas unidades de emergência seria 0 atendimento resolutivo nas unidades de atenção primária à saúde e o esclarecimento da população e dos profissionais sobre a real finalidade dos serviços de urgência e emergência. ${ }^{2}$

\section{CONCLUSÃO}

Este estudo, ao analisar o dimensionamento dos profissionais de enfermagem, buscou evidenciar a importância desse processo como um instrumento gerencial para avaliar, planejar e distribuir o quantitativo mínimo de profissionais de enfermagem necessários para prestar assistência de qualidade e segura no pronto-socorro. Houve predomínio de pacientes adultos e idosos e do sexo feminino.

Verificou-se que havia uma média diária de 2,8 enfermeiros e 15,1 técnicos de enfermagem no quadro de pessoal existente na unidade. No entanto, para o quadro recomendado, sem considerar o quadro de pessoal para cobertura de faltas previstas e não previstas eram necessários 8,6 enfermeiros e 16,5 técnicos e auxiliares de enfermagem para atender a demanda da unidade nas 24 horas.

Ao aplicar o SCP foi possível verificar o predomínio de pacientes classificados como cuidados mínimos. Isto remete à possibilidade de que as diferenças entre a lógica da 


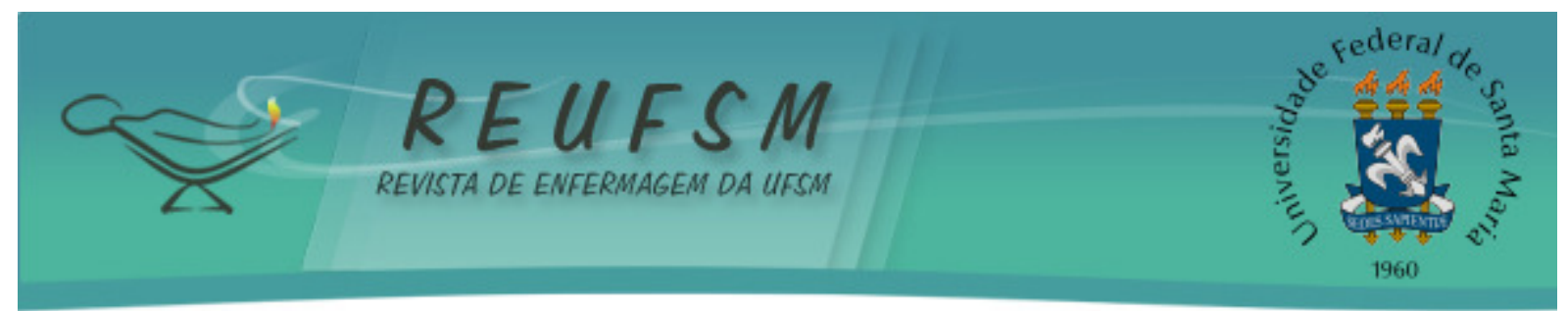

população e a organização do sistema de saúde, além de outros fatores, determinam a utilização inadequada da atenção terciária, gerando número expressivo de atendimentos que poderiam ser resolvidos na atenção primária ou secundária à saúde.

Portanto, para que as pessoas que requerem atendimento com menor densidade tecnológica possam ser atendidas na atenção primária à saúde, faz-se necessário rever a gestão do modelo de atenção para atender as demandas dos prontos-socorros. Essa problemática é agravada pelo quantitativo reduzido de profissionais de enfermagem no setor para prestar assistência com qualidade à alta demanda. Quando os recursos são limitados, dificilmente todas as necessidades são atendidas e isso gera riscos para a segurança do paciente, bem como à saúde ocupacional.

Diante do exposto, a classificação dos pacientes permite sustentar o planejamento das ações de enfermagem e mostra-se necessária para subsidiar o dimensionamento dos recursos humanos, contribuindo para a realização da assistência ao paciente com maior qualidade e segurança.

Acredita-se que os resultados do estudo permitem que os profissionais assistenciais e gerenciais atuantes no pronto-socorro e gestores, conhecerem o perfil dos pacientes atendidos e a real necessidade de profissionais para assisti-los e instigar a necessidade de planejar estratégias de identificação e acolhimento de pacientes que necessitam de atendimento imediato, objetivando a qualidade da assistência e a organização do serviço.

Salienta-se, contudo, que existem limitações da pesquisa pelo fato da mesma ter sido desenvolvida desconsiderando a organização do serviço e estrutura física.

\section{REFERÊNCIAS}

1. Brasil. Ministério da Saúde. Política nacional de atenção às urgências. Brasília: Ministério da Saúde; 2006.

2. Azevedo ALCS, Pereira AP, Lemos C, Coelho MF, Chaves LDP. Organização de serviços de emergência hospitalar: uma revisão integrativa de pesquisas. Rev Eletrônica Enferm [Internet]. 2010 [acesso em 2012 dez 12];12(4):736-45. Disponível em: http://dx.doi.org/10.5216/ree.v12i4.6585.

3. Brasil. Ministério da Saúde. Secretaria de Atenção à Saúde. Departamento de Atenção Especializada. Implementação da rede de atenção às urgências/emergências - RUE. Brasília: Ministério da Saúde, 2011. (Nota técnica; 14).

4. Ohara R, Melo MRAC, Laus AM. Caracterização do perfil assistencial dos pacientes adultos de um pronto socorro. Rev Bras Enferm 2010;63(5):749-54.

5. Brasil. Ministério da Saúde. Portaria $n^{\circ} 4.279$, de 30 de dezembro de 2010. Estabelece diretrizes para a organização da Rede de Atenção à Saúde no âmbito do Sistema Único de Saúde (SUS) [Internet]. [acesso em 2013 mar 20]. Disponível em: http://bvsms.saude.gov.br/bvs/saudelegis/gm/2010/prt4279_30_12_2010.html.

6. Silva DS, Bernardes A, Gabriel CS, Rocha FLR, Caldana G. A liderança do enfermeiro no contexto dos serviços de urgência e emergência. Rev Eletrônica Enferm [Internet]. 2014 jan/mar;16(1):211-9. Disponível em: http://dx.doi.org/10.5216/ree.v16i1.19615.

7. Kurgant P. Gerenciamento de enfermagem. $2^{\mathrm{a}}$ ed. Rio de Janeiro: Guanabara Koogan; 2010.

8. Conselho Federal de Enfermagem (COFEN). Resolução COFEN $n^{\circ} 293$, de 21 de setembro de 2004. Fixa e estabelece parâmetros para o dimensionamento do quadro de profissionais 


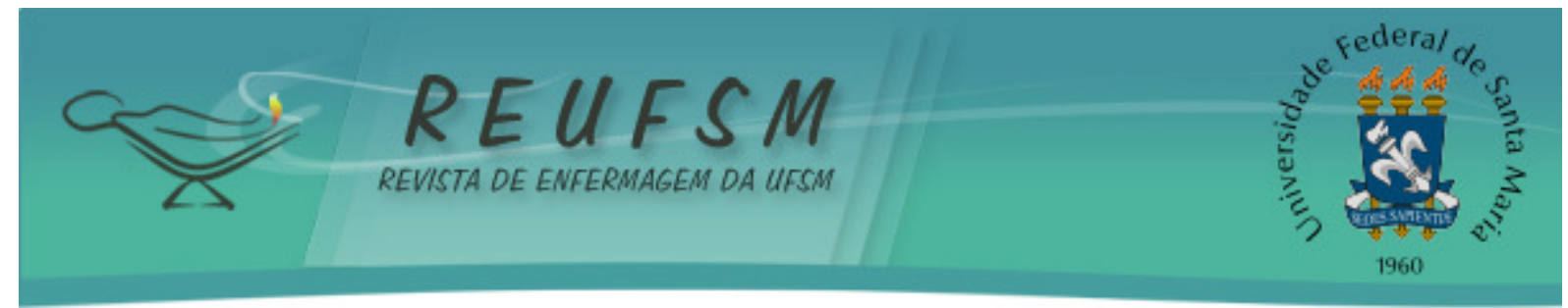

de enfermagem nas unidades assistenciais das instituições de saúde e assemelhados [Internet]. [acesso em 2012 dez 12]. Disponível em: http://novo.portalcofen.gov.br/resoluo-cofen-2932004_4329.html.

9. Fugulin FMT, Silva SH, Shimizu HE, Campos FPF. Implantação do sistema de classificação de pacientes na unidade de clínica médica do Hospital Universitário da Universidade de São Paulo. Rev Med Hosp Univ. 1994;4(1/2):63-8.

10. Dini AP. Sistema de classificação de pacientes pediátricos: construção e validação de instrumento [dissertação]. Campinas: Faculdade de Ciências Médicas/UNICAMP; 2007. $170 \mathrm{p}$.

11. Conselho Federal de Enfermagem (COFEN). Lei $n^{\circ} 7.498$, de 25 de junho de 1986. Dispõe sobre a regulamentação do exercício da Enfermagem e dá outras providências [Internet]. 1986 [acesso em 2013 mar 05]. Disponível em: http://novo.portalcofen.gov.br/lei-n-749886-de-25-de-junho-de-1986_4161.html.

12. Versa GLGS, Inoue KC, Nicola AL, Matsuda LM. Influência do dimensionamento da equipe de enfermagem na qualidade do cuidado ao paciente crítico. Texto \& Contexto Enferm. 2011;20(4):796-802.

13. Fassini $P$, Hahn GV. Riscos à segurança do paciente em unidade de internação hospitalar: concepções da equipe de enfermagem. Rev Enferm UFSM 2012;2(2):290-9.

14. Montezelli JH, Peres AM, Bernardino E. Demandas institucionais e de cuidado no gerenciamento de enfermeiros em um pronto socorro. Rev Bras Enferm. 2011; 64(2):348-54.

15. Garcia EA, Fugulin FMT. Nurses' work time distribution at the emergency service. Rev Esc Enferm USP. 2010;44(4):1032-8.

Data de recebimento: $24 / 01 / 2015$

Data de aceite: $11 / 06 / 2015$

Contato do autor responsável: Ana Cristina Geiss Casarolli

Endereço postal: Rua Elpídio Menezes $n^{\circ} 21$ Apto. 106, Bairro Camobi, Santa Maria, Rio Grande do Sul, Brasil, CEP 97105-110

E-mail: anacasarolli@hotmail.com 